\title{
Chronic Suppurative Osteomyelitis of Mandible: A Case Report
}

\author{
Hemant Mehra, MDS ${ }^{1}$ Sumit Gupta, MDS ${ }^{1} \quad$ Hemant Gupta, MDS ${ }^{1} \quad$ V. Sinha, MDS ${ }^{1}$ Jasmeet Singh, MDS ${ }^{1}$ \\ 1 Department of Oral and Maxillofacial Surgery, Babu Banarasi Das \\ College of Dental Surgery, Lucknow, Uttar Pradesh, India \\ Craniomaxillofac Trauma Reconstruction 2013;6:197-200

\begin{abstract}
Address for correspondence Hemant Mehra, MDS, Babu Banarasi Das College of Dental Surgery-Oral and Maxillofacial Surgery, Akhilesh Das Nagar, Lucknow, Uttar Pradesh, India (e-mail: hemantmehra121@gmail.com).
\end{abstract}

\begin{abstract}
Keywords

- osteomyelitis

- sequestrum

- surgical debridement

- reconstruction plate

- pathologic fracture

Osteomyelitis is an inflammatory disease of the bone that usually begins as an infection of the medullary cavity, rapidly involves the haversian system, and quickly extends to the periosteum of the area. It develops in the jaws after a chronic odontogenic infection or for a variety of other reasons such as trauma, inadequate treatment of fracture, or irradiation to the mandible. When antimicrobial agents or drainage prove unsuccessful, acute osteomyelitis may become chronic. Conventional radiography, culture, bone biopsy, radioisotope bone scan, laser Doppler flowmetry, computed tomography, and magnetic resonance imaging are used for its diagnosis. We present a case of chronic suppurative osteomyelitis associated with a draining extraoral sinus, which was successfully treated with surgical debridement and stabilization with a 10-hole reconstruction plate and bicortical screws using AO principles.
\end{abstract}

\section{Case Report}

A 48-year-old man reported to the Department of Oral and Maxillofacial Surgery, BBD College of Dental Sciences, Lucknow with an extraorally draining sinus in the left anteroinferior border of mandible and a foul odor from the oral cavity for 4.5 months. The patient also complained of paresthesia of the left lower lip for the previous 1.5 months.

The patient reported having pain in the lower left back of the jaw 4.5 months previously. He applied peppermint oil in the region of a carious tooth intraorally and subsequently developed an extraorally draining sinus at the left inferior border of mandible. The patient visited a local dentist who extracted the left mandibular second premolar and first and second molars; following the extractions, pain decreased but the draining sinus and dull continuous pain persisted. As the condition did not improve, the patient presented to our department for definitive treatment (-Fig. 1).

Preoperative routine investigations were performed. A panoramic radiograph was taken, which revealed bifocal radiolucent areas extending between left mandibular first premolar and third molar (-Fig. 2). Based on clinical and radiographic presentation, a diagnosis of chronic osteomyelitis was made.

The treatment plan consisted of surgical debridement and stabilization with reconstruction plate using AO principles with three bicortical screws on both sides.

Under general anesthesia, the lesion was approached extraorally using a submandibular incision. On exposure, destruction of the lingual cortex of the mandible was seen even though the buccal cortex was spared. Surgical debridement was done and stabilization with a 10-hole reconstruction plate used AO principles; three bicortical screws on both sides were used for fixation to prevent pathologic fracture of the weakened mandible after thorough debridement during sequestrectomy (-Fig. 3). The entire sinus tract was excised and the wound was closed in three layers. Resected tissue was sent for histopathologic examination. Root tips of no. 30 site was also removed as it could have acted as a nidus of infection and caused recurrence of the condition.

Postoperative care included intravenous antibiotics (ceftriaxone $1 \mathrm{~g}$ twice a day and metronidazole $100 \mathrm{~mL}$ three times day, based on culture sensitivity reports) for 5 days, followed by oral dosage up to 2 weeks with analgesics, received

July 30, 2012

accepted

August 20, 2012

published online

May 31, 2013
Copyright $\odot 2013$ by Thieme Medical Publishers, Inc., 333 Seventh Avenue, New York, NY 10001, USA. Tel: +1(212) 584-4662.
DOI http://dx.doi.org/ 10.1055/s-0033-1343781. ISSN 1943-3875. 


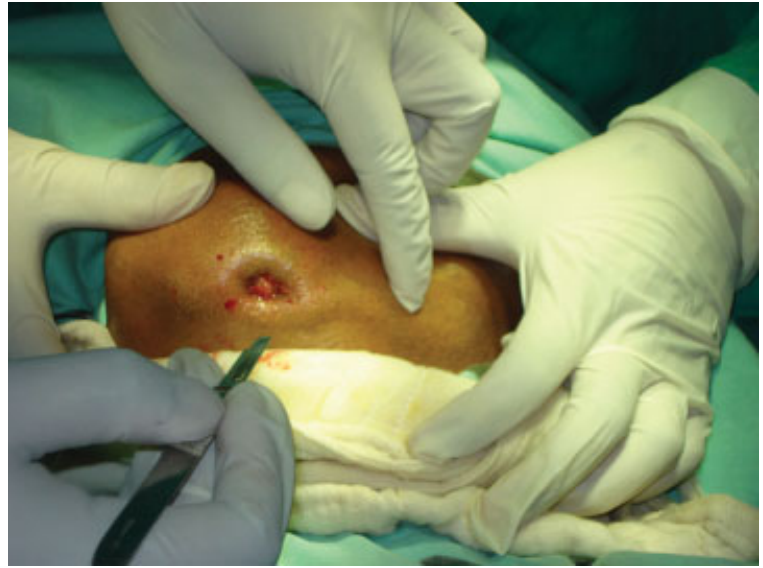

Figure 1 Extra oral photograph showing extraoral draining sinus.

adequate hydration, and rest. The wound healed uneventfully without any postoperative complications. Panoramic radiographs were taken at 2-month intervals ( - Fig. $\mathbf{4}$ and - Fig. 5). Panoramic radiograph 6 months postoperatively showed considerable resolution. Paresthesia of the left side of lower lip resolved completely by 6 months postoperatively (-Fig. 6). Absence of any clinical signs of infection and sequential postoperative radiographs at different times demonstrated clinical success, showing resolution of pathology by decreased radiolucency indicating osteogenesis.

\section{Discussion}

Chronic osteomyelitis is a bone disease that is characterized by inflammatory processes, including necrosis of mineralized and marrow tissues, suppuration, resorption, sclerosis, and hyperplasia. ${ }^{1,2}$ The primary cause of chronic osteomyelitis is usually microbiologic, and it results from an odontogenic infection, postextraction complication, inadequate removal of necrotic bone, early termination of antibiotic therapy, inappropriate selection of antibiotics, diagnostic failure, trauma, inadequate treatment for fracture, or irradiation to the mandible. ${ }^{3,4}$ Probable cause of disease in our case was odontogenic infection in relation to left lower posterior teeth.

Clinical findings in chronic mandibular osteomyelitis include local pain, fever, swelling, purulent discharge, intraoral and skin fistula, unhealed soft tissue in the oral cavity, neuropalsy in the involved area, pathologic fracture, and

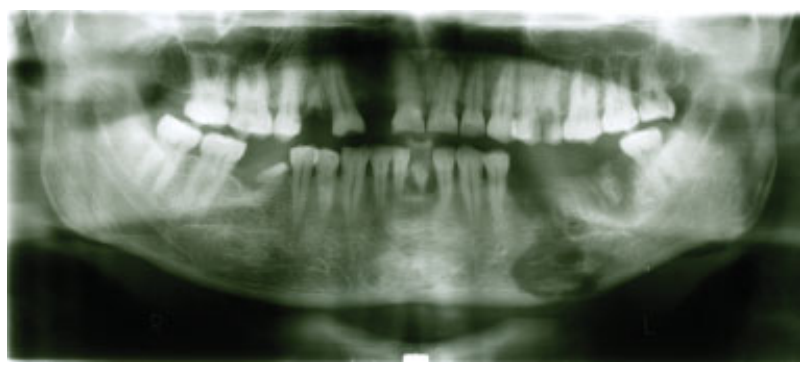

Figure 2 Preoperative panoramic radiograph.

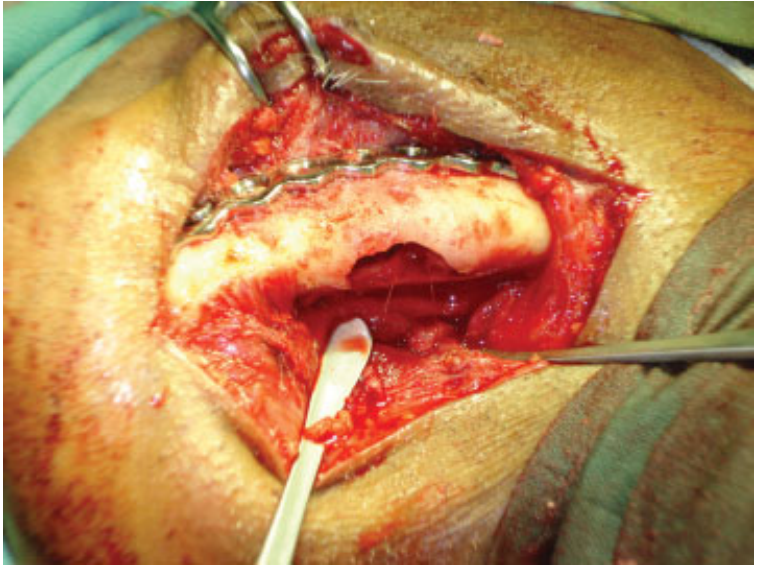

Figure 3 Bony defect on the lingual side after debridement and buttressing with reconstruction plate.

trismus. Our patient presented to us with dull aching pain, paresthesia in left lower lip region, and an extraorally draining sinus in the left anteroinferior border of mandible and foul odor.

Radiologic examination discloses radiolucent areas, bony destruction, and sequestrum formation. ${ }^{1}$ The presence of sequestra and laminations of periosteal new bone are useful distinguishing features of osteomyelitis. ${ }^{5}$ Positive radiographic findings are usually delayed or secondary, except in cases with an associated fracture. ${ }^{6}$ Computed tomography (CT) gives a more definitive picture of tissue calcification. CT findings included sclerosis and defect in the trabecular bone. Changes of bone width and thickening of the cortical plate are accompanied with longer disease duration. The extent of the diseased area is linearly correlated with the duration of symptoms. ${ }^{7}$ Radioisotope bone scanning reveals strong uptake $^{8}$ and can be used to identify occult areas of involvement. Positron emission tomography is a better modality for the delineation of areas of different metabolic activity. ${ }^{6}$ Scintigraphy with bone-seeking radiopharmaceuticals is used when multifocal systemic disease is suspected. ${ }^{9}$ Magnetic resonance imaging in short inversion time inversion recovery sequence is highly effective for the evaluation of bone marrow and surrounding soft tissue in terms of the detection of osteomyelitis in the mandible and the identification of inflammation spreading to soft tissue. ${ }^{10}$ Magnetic resonance imaging shows larger areas of abnormality than plain radiography or $\mathrm{CT} .{ }^{11}$ In the present case, an orthopantomogram was taken for radiologic examination, which revealed bifocal radiolucent areas extending between the left mandibular first premolar and the third molar. It also showed presence of radiopaque involucrum and radiolucent sequestra in the left mandibular body region, suggestive of osteomyelitis.

A diagnosis of osteomyelitis is based on the presence of painful sequestra and suppurative areas in the tooth-bearing jawbone that are unresponsive to debridement and conservative therapy. ${ }^{6}$ Tissue specimens should be cultured for the presence of microorganisms. Once soft tissue and bone specimens have been obtained, they must be sent to the microbiology laboratory immediately to identify the 


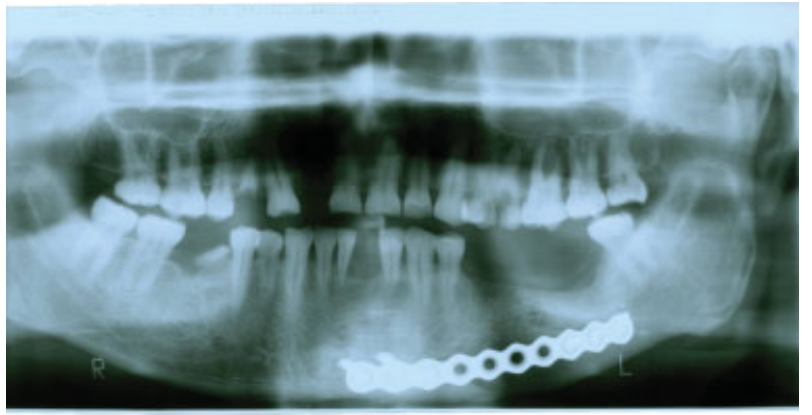

Figure 4 Two months postoperative panoramic radiograph.

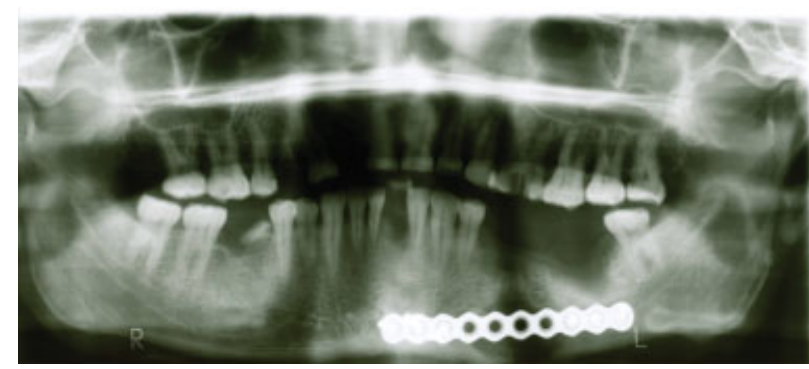

Figure 5 Four months postoperative panoramic radiograph.

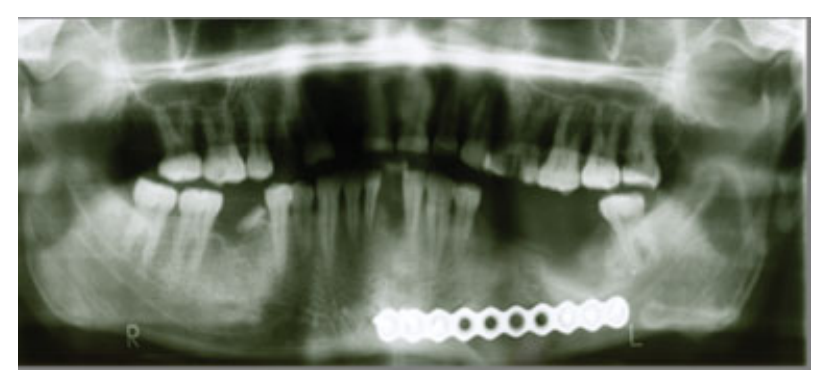

Figure 6 Six months postoperative panoramic radiograph.

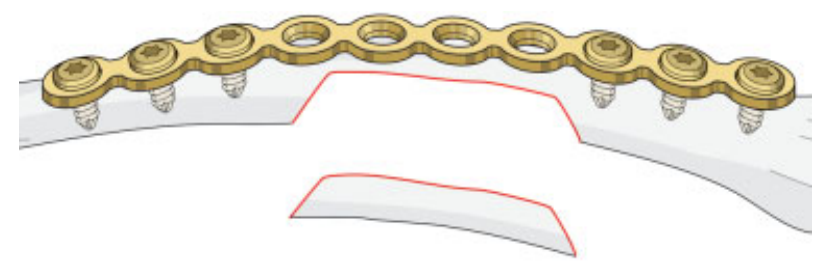

Figure 7 Reconstruction plate used to abut the weakened section of the bone following removal of pathology using $\mathrm{AO}$ principles of rigid internal fixation with three screws each on either side.

microorganisms. ${ }^{12}$ Histologically, an increased number of osteoblasts, thickened bony trabecula, and fibrous marrow replacement are found. Pathologic bone remodeling and the presence of chronic inflammatory cells are often cited as indicators of osteomyelitis. ${ }^{13}$ Pathogenic organisms usually implicated in chronic mandibular osteomyelitis are normal oral flora, Staphylococcus aureus and aerobic gram-negative bacilli. ${ }^{14}$ In our case, the clinical and radiographic diagnosis was confirmed by histologic examination of the specimen. The histopathology revealed fibrocellular connective tissue stroma with necrotic bony trabeculae with empty lacunae. Dense fibrous tissue with inflammatory cells, including lymphocytes and plasma cells, confirmed chronic osteomyelitis.

The differential diagnosis of these lesions includes Paget disease, hypercementosis, fibrous dysplasia, early stage malignant bone tumor, osteogenic sarcoma, and fibrous dysplasia., 5

The treatment of chronic mandibular osteomyelitis involves thorough surgical debridement and prolonged antimicrobial therapy as was done in our case. Additionally a 10hole reconstruction plate was applied with three bicortical screws each on both sides of the defect to buttress the weakened mandible in the operated area. This was done following the principles of $\mathrm{AO}$ as suggested by Speissel et al. Placement of a reconstruction plate held with $10-\mathrm{mm}$ screws holds the mandible weakened due to the surgical debridement and causes a buttressing effect, reducing chance of pathologic fracture (-Fig. 7). In addition, appropriate fluid therapy, nutrient intake, antibiotic containing acrylic beads, microvascular grafts, and sufficient rest may be useful. ${ }^{14,16,17}$ According to Bernier S. et al, surgical therapy consists of sequestrectomy or decortication with saucerization of the affected bone, as well as the removal of necrotic tissues, broken teeth, and roots. ${ }^{17,18}$ Van Merkesteyn and Bakker ${ }^{19}$ reported that a combined antibiotic and surgical approach is the treatment of choice in chronic suppurative osteomyelitis. Bamberger $^{2}$ reported that at least 4 weeks of antibiotic therapy are required after surgery, whereas Marx ${ }^{12}$ stated that there was a need for at least 2 weeks of antibiotic therapy after surgery. Montonen et al ${ }^{17}$ described possible causes of failure that include insufficiently radical surgery and the retention of devitalized teeth in the decorticated area. However, in the more refractory forms and in patients in whom decortication and antibiotic therapy have failed, hyperbaric oxygen treatment in combination with antibiotics and surgery is indicated to produce microvascular neoangiogenesis to increase reperfusion. ${ }^{6}$

Our case showed significant radiographic resolution of the postoperative bony defect in 6 months time, which can be attributed to total elimination of infectious process from the region postoperatively. No pathologic fracture was seen on subsequent radiographs, which can be attributed to the buttressing effect of rigid fixation and stabilization with reconstruction plate.

\section{Conclusion}

The present case shows that surgical debridement is a definitive method of treating chronic suppurative osteomyelitis of the mandible, with favorable clinical/radiologic results and postoperative function. Stabilization of bone by rigid internal fixation decreases the possibility of pathologic fracture and improves prognosis.

\section{References}

1 Goaz PW, White SC. Infection and inflammation of the jaws and facial bones. In: Goaz PN, White SC, eds. Oral Radiology Principles and Interpretation. St. Louis, MO: Mosby; 1994:386-395 
2 Bamberger DM. Osteomyelitis. A commonsense approach to antibiotic and surgical treatment. Postgrad Med 1993;94:177-182, 184

3 Daramola JO, Ajagbe HA. Chronic osteomyelitis of the mandible in adults: a clinical study of 34 cases. Br J Oral Surg 1982;20:58-62

4 Stafne EC. Infections or the jaws. In: Cibilisco JA, eds. Stafne's Oral Radiographic Diagnosis. Philadelphia, PA: Saunders; 1985:86

5 Petrikowski CG, Pharoah MJ, Lee L, Grace MG. Radiographic differentiation of osteogenic sarcoma, osteomyelitis, and fibrous dysplasia of the jaws. Oral Surg Oral Med Oral Pathol Oral Radiol Endod 1995;80:744-750

6 I-Hudson JW. Osteomyelitis of the jaws: a 50-year perspective. J Oral Maxillofac Surg 1993;51:1294-301

7 Ida M, Watanabe H, Tetsumura A, Kurabayashi T. CT findings as a significant predictive factor for the curability of mandibular osteomyelitis: multivariate analysis. Dentomaxillofac Radiol 2005;34:86-90

8 Van Merkesteyn JP, Groot RH, Bras J, Bakker DJ. Diffuse sclerosing osteomyelitis of the mandible: clinical radiographic and histologic findings in twenty-seven patients. J Oral Maxillofac Surg 1988;46: 825-829

9 Graffman S, Rangne A. Scintigraphy in diagnosis of osteomyelitis of the jaws. Int J Oral Surg 1977;6:247-250

10 Lee K, Kaneda T, Mori S, Minami M, Motohashi J, Yamashiro M. Magnetic resonance imaging of normal and osteomyelitis in the mandible: assessment of short inversion time inversion recovery sequence. Oral Surg Oral Med Oral Pathol Oral Radiol Endod 2003;96:499-507
11 Kaneda T, Minami M, Ozawa K, et al. Magnetic resonance imaging of osteomyelitis in the mandible. Comparative study with other radiologic modalities. Oral Surg Oral Med Oral Pathol Oral Radiol Endod 1995;79:634-640

12 Marx RE. Chronic osteomyelitis of the jaws. Oral Maxillofac Surg Clin North Am 1991;3:367-381

13 Van Merkesteyn JP, Groot RH, van den Akker HP, Bakker DJ, Borgmeijer-Hoelen AM. Treatment of chronic suppurative osteomyelitis of the mandible. Int J Oral Maxillofac Surg 1997;26: 450-454

14 Gentry LO. Osteomyelitis: options for diagnosis and management. J Antimicrob Chemother 1988;21:15-31

15 Ylikontiola L, Altonen M, Uhari M, et al. Chronic sclerosing osteomyelitis of the mandible in monozygotic twins. Int. J Oral Maxillofac Surg 1994;23:359-362

16 Rohlin M. Diagnostic value of bone scintigraphy in osteomyelitis of the mandible. Oral Surg Oral Med Oral Pathol 1993;75: 650-657

17 Montonen M, Iizuka T, Hallikainen D, Lindqvist C. Decortication in the treatment of diffuse sclerosing osteomyelitis of the mandible. Retrospective analysis of 41 cases between 1969 and 1990. Oral Surg Oral Med Oral Pathol 1993;75:5-11

18 Bernier S, Clermont S, Maranda G, Turcotte JY. Osteomyelitis of the jaws. J Can Dent Assoc 1995;61:441-442, 445-448

19 Van Merkesteyn JP, Bakker DJ, Van der Waal I, et al. Hyperbaric oxygen treatment of chronic osteomyelitis of the jaws. Int J Oral Surg 1984;13:386-395 